\title{
Watching My Mind Unfold vs. Yours: An fMRI Study Using a Novel Camera Technology to Examine Neural Differences in Self-Projection of Self vs. Other Perspectives
}

\author{
Peggy L. St. Jacques ${ }^{1,2}$, Martin A. Conway ${ }^{3}$, Matthew W. Lowder ${ }^{1}$, and Roberto Cabeza ${ }^{1,2}$ \\ ${ }^{1}$ Center for Cognitive Neuroscience, Duke University, Durham, NC, 27708, USA \\ 2Department of Psychology and Neuroscience, Duke University, Durham, NC, 27708, USA \\ ${ }^{3}$ Institute of Psychological Sciences, University of Leeds, Leeds, LS2 9JT, UK
}

\section{Abstract}

Self-projection, the capacity to re-experience the personal past and to mentally infer another person's perspective, has been linked to the medial prefrontal cortex (mPFC). In particular, ventral mPFC is associated with inferences about one's own self, whereas dorsal mPFC is associated with inferences about another individual. In the present functional MRI study we examined selfprojection using a novel camera technology, which employs a sensor and timer to automatically take hundreds of photographs when worn, in order to create dynamic visuospatial cues taken from a first-person perspective. This allowed us to ask participants to self-project into the personal past or into the life of another person. We predicted that self-projection to the personal past would elicit greater activity in the ventral $\mathrm{mPFC}$, whereas self-projection of another perspective would rely on dorsal $\mathrm{mPFC}$. There were three main findings supporting this prediction. First, we found that self-projection to the personal past recruited greater ventral $\mathrm{mPFC}$, whereas observing another person's perspective recruited dorsal mPFC. Second, activity in ventral vs. dorsal mPFC was sensitive to parametric modulation on each trial by the ability to relive the personal past or to understand another's perspective, respectively. Third, task-related functional connectivity analysis revealed that ventral mPFC contributed to the medial temporal lobe network linked to memory processes, whereas dorsal mPFC contributed to the frontoparietal network linked to controlled processes. In sum, these results suggest that ventral-dorsal subregions of the anterior midline are functionally dissociable and may differentially contribute to self-projection of self vs. other.

Self-projection is the capacity that allows us to shift our perspective from the present moment to alternative temporal and mental locations (Buckner \& Carroll, 2007; Mitchell, 2009; Suddendorf \& Corballis, 2007). The neural correlates supporting temporal simulations of the personal past during autobiographical memory (AM) retrieval overlap with those supporting mental simulations of another person's perspective during Theory of Mind (ToM) tasks (for metaanalysis see Spreng, Mar, \& Kim, 2009). However, few functional neuroimaging studies have manipulated self-projection within the same individual (although see Rabin, Gilboa, Stuss, Mar, \& Rosenbaum, 2009; Spreng \& Grady, 2009) while also using naturalistic stimuli. In the present functional MRI (fMRI) study we examined temporal and mental forms of self-projection using a novel camera technology to create dynamic visuospatial cues which provided the opportunity to step into the personal past or another individual's perspective.

Corresponding Author: Peggy L. St. Jacques, Center for Cognitive Neuroscience, Duke University, Box 90999, Durham, NC 27708, USA, Phone: (919) 668-2299, Fax: (919) 681-0815, peggy.st.jacques@ duke.edu. 
Functional neuroimaging studies have linked self-projection to the medial prefrontal cortex (mPFC; Mitchell, 2009). The medial PFC is involved in abstract forms of mentalizing (Amodio \& Frith, 2006; Gallagher \& Frith, 2003) such as integrating social information about the stable dispositions of others and the self across time (Van Overwalle, 2009). Subregions within the mPFC, however, may differentially contribute to self-projection. Ventral mPFC is sensitive to the ability to re-experience the self in time during AM (Levine et al., 2004; Maguire \& Mummery, 1999), and focal lesions that overlap here impair the subjective experience of re-experiencing the personal past (Wheeler \& Stuss, 2003). Moreover, resting-state functional connectivity has shown that subregions of the mPFC comprise separable networks. Ventral mPFC is linked to the hippocampus and other regions associated with the medial temporal lobe (MTL) network (Kahn, Andrews-Hanna, Vincent, Snyder, \&Buckner, 2008; Vincent et al., 2007), a system of brain regions important for internally directed processes, such as memory. In contrast, dorsal mPFC is linked to dorsolateral PFC and lateral parietal cortices associated with the frontoparietal network (Vincent, Kahn, Snyder, Raichle, \& Buckner, 2008), a system of brain regions associated with controlled processes. Ventral $\mathrm{mPFC}$ has been associated with inferences about one's own self and dorsal mPFC with inferences about another individual (Krueger, Barbey, \& Grafman, 2009; Mitchell, 2009; Van Overwalle, 2009). However, there is considerable debate regarding the functional specialization of the $\mathrm{mPFC}$ with respect to self versus other processing (Northoff et al., 2006).

The goal of the present fMRI study was to examine self-projection of self vs. other elicited by naturalistic stimuli within the same individuals by employing a novel camera technology. We tested three main predictions regarding the role of mPFC, which were based on the evidence regarding ventral and dorsal subregions in self versus other processing (Krueger, et al., 2009; Mitchell, 2009; Van Overwalle, 2009). First, ventral mPFC will be preferentially recruited during self-projection of self, whereas dorsal $\mathrm{mPFC}$ will be recruited to a greater extent for self-projection of other. Second, ventral vs. dorsal mPFC will be sensitive to variability in the ability to re-experience the personal past and to understand another's perspective. Third, ventral vs. dorsal $\mathrm{mPFC}$ will be functionally connected to separable neural networks contributing to self-projection of self vs. other.

\section{Methods}

\section{Participants}

There were twenty-three participants ( 12 females; Mean Age $=23.7, S D=3.6$ ) who were healthy, right-handed, and without history of neurological or psychiatric episodes. Participants gave written informed consent for a protocol approved by the Duke University Institutional Review Board.

\section{Procedure}

\section{SenseCam-SenseCam}

(http://research.microsoft.com/en-us/um/cambridge/projects/sensecam/) is a small wearable digital camera that has electronic sensors (e.g., light, heat, motion) that can automatically and silently trigger thousands of photographs in a single day (see Figure 1A). This differs considerably from the normal way in which we can use a camera to generate retrieval cues (Cabeza et al., 2004; St Jacques, Rubin, Labar, \& Cabeza, 2008), because it does not disrupt the ongoing experience of events through the act of taking a photograph (also see Levine, et al., 2004). Several photographs taken from a particular event (e.g., eating ice-cream; see Figure 1B) can be consecutively viewed to create a dynamic visuospatial cue (http://www.youtube.com/watch?v=sr1i-sICafs). The SenseCam lens also maximizes the field of view to better capture the perspective of the wearer by incorporating a wide-angle 
(fish-eye) lens. In sum, the SenseCam captures dynamic images taken from the first person perspective, which provide highly effective cues for self-projection to alternative self vs. other perspectives.

Prospective Collection-Retrieval cues were prospectively collected, such that participants were asked to keep a record of their lives prior to the scanning session by wearing the SenseCam. Participants wore the SenseCam for 6 days and kept a schedule of each day's activities to be used to parse the SenseCam images into events. The daily schedule was recorded at the end of each day along with a unique identifier to distinguish that particular day from others (e.g., "Today was the day I had lunch with Ben"). Participants were instructed to write about 10-15 brief sentences, one for each major event during the day (e.g., "Had breakfast", "went to the grocery store ...") which were used to segregate the images into events.

Cue Selection-Three days were randomly assigned to elicit self-projection of one's own self (SPS). The content of the remaining three days was used for an analysis that was not the main focus of the present investigation (St Jacques, Conway, \& Cabeza, 2010). For each day, 12 events were selected to be tested in the scanner. To elicit self-projection of other perspectives (SPO), images were collected by three volunteers who wore a SenseCam in locations at least 100 miles away from Duke University and whose lifestyles differed from the participants. Images in the SPO condition depicted events that would have been familiar to the participants (e.g., going to the going to the grocery store, eating at a restaurant, going to Wal-Mart, renting a movie from Blockbuster, etc.) but which were not self-relevant, thus minimizing the likelihood that the other-perspective condition would include pictures of self-relevant people, places, and activities, which might inadvertently trigger AMs. Debriefing following the scanning session indicated that the SPO images were familiar to many of the participants, but did not trigger personal memories. The SPO images were also carefully selected to be similar to the SPS images (e.g., indoor/outdoor, time of day, etc.). Images in both conditions were selected to ensure good picture quality.

FMRI Scanning-The scanning session took place one week following the last day the SenseCam was worn (mean length of delay $=8$ days, $S D=1.2$ ). There were a total of nine fMRI runs blocked by condition and presented in an alternating order (i.e., ABCABCABC), counterbalanced across participants based on a Latin square design. Three of the separate runs consisted of another condition, which was included for a separate analysis. The structure of the remaining six runs was similar in each condition (see Figure 1C). Each of these runs began with a 10 -second title screen (i.e., "Today was the day I...") and consisted of 12 cues presented in chronological order from that day, for a total of 36 events per condition across 3 runs. Cues were presented for 20 seconds, and participants were instructed to recall the events depicted from their own perspective (SCS) or to understand the events being depicted from another person's perspective (SCO). The cues in each condition consisted of 40 SenseCam pictures depicting a single event and presented at a rate of two pictures per second.

Following each cue presentation, participants indicated their subjective experience. In the SPS condition participants rated the subjective experience of recollection, reliving, which refers to how much they were able to re-experience the event depicted as if it were happening right now or as if they were mentally traveling back to the time when the event occurred. It is important to note that reliving is similar to other subjective measures of recollection, such as the remember/know paradigm (for a review see Yonelinas, 2002). For example, in the remember/know paradigm, participants are asked to use introspection to classify items as recollected (vivid re-experiencing of the original event and its context) or merely familiar. Although introspection has its limitations, the results of hundreds of 
remember/know studies are highly consistent with findings of hundreds of studies using objective measures of recollection, such as source memory (Yonelinas, 2002). However, there are some critical differences between the reliving scale and remember/know paradigm, which make the reliving scale a better measure for AM. First, the reliving scale could be considered a better subjective measure of recollection in AM than remember/know because it does not require the assumption of a dual-process model (Wixted, 2007; Yonelinas, 2002). Second, reliving is a better predictor of recollection in AMs compared to the remember/ know scale, which is a better predictor of confidence in AMs (Rubin, Burt, \& Fifield, 2003; Rubin, Schrauf, \& Greenberg, 2004). In the SPO condition participants were asked to indicate the amount of understanding of the other person's perspective. While taking another person's perspective, participants were instructed to try to understand what was happening, where the event was taking place, and why the event was occurring. Ratings were conducted on an 8-point scale from low to high, and were self-paced (up to 6 seconds). Following a response, a fixation cross was presented for a jittered interval between 4 and 8 seconds plus any remaining time from the response period.

\section{fMRI Methods}

Image Acquisition-Scanning was conducted using a 4T GE magnet. Anatomical scanning included a T1-weighted sagittal localizer series and 3D fast spoiled gradient echo recalled (SPGR) structural images were acquired in the coronal plane $\left(256^{2}\right.$ matrix, $\mathrm{TR}=$ $12.3 \mathrm{~ms}, \mathrm{TE}=5.4 \mathrm{~ms}$, flip angle $=20^{\circ}, \mathrm{FOV}=240,68$ slices, $1.9 \mathrm{~mm}$ slice thickness). Coplanar functional images were acquired using an inverse spiral sequence $\left(64^{2}\right.$ image matrix, $\mathrm{TR}=2000 \mathrm{~ms}, \mathrm{TE}=6 \mathrm{~ms}, \mathrm{FOV}=240$, flip angle $=60^{\circ}$, 34 slices, $3.8 \mathrm{~mm}$ slice thickness).

fMRI Analyses-Image processing and analyses were performed using Statistical Parametric Mapping software in Matlab (SPM5; Wellcome Department of Imaging Neuroscience). Functional images were corrected for slice acquisition order, realigned to correct for motion artifacts, spatially normalized to a standard stereotactic space, and spatially smoothed using an 8-mm isotropic Gaussian kernel. Coordinates are reported in Talariach space using a transformation from the Montreal Neurological Institute coordinates (Brett, Christoff, Cusack, \& Lancaster, 2001)

Self-Projection of Self vs. Other-To examine activation differences between self vs. other self-projection during the presentation of the SenseCam images, we used a Finite Impulse Response (FIR) basis function. The FIR approach allowed us to examine potential activation differences without assuming a particular canonical hemodynamic response function, and thus, was appropriate for the complex and temporally protracted processes elicited in the present study. The FIR model included 16 regressors of peristimulus time bins of $2 \mathrm{~s}$ duration (equal to the TR) for each condition, yielding estimates of fMRI signal change across the entire trial period (SenseCam presentation and Rating).

We conducted a Condition (SPS, SPO) $\times$ Time ( 0 to 20s) ANOVA implemented in SPM5 in order to isolate activation differences in the self vs. other conditions across the FIR timepoints associated with the presentation of the SenseCam images. We examined the Main Effect of Condition at an FDR corrected threshold of $\mathrm{P}=.05$ using a 2 voxel extent threshold, and inclusively masked with the effect of interest (SPS > SPO or SPO > SPS) at P $=.05$ to determine the direction of the effect. An extent threshold of 2 voxels was chosen here because the ROI approach combined with a corrected threshold was considered very conservative. Further, we took a region of interest (ROI) approach to examine brain regions associated with self vs. other self-projection in the present study based on a previous quantitative metaanalysis which generated activation likelihood estimation maps 
corresponding to statistically significant concordance of activated voxels in $19 \mathrm{AM}$ and 50 ToM studies (Spreng, et al., 2009).

Parametric Modulation by Behavior-To examine the neural correlates associated with self vs. other self-projection that was sensitive to online behavioral responses we employed a parametric approach. To identify increases in activity as a function of increasing behavioral responses on each trial, we created a GLM in which temporal vs. mental selfprojection was modulated by reliving and understanding using the first-order parametric modulation option integrated in SPM5. Subsequently, random-effects analyses were performed on the parameter estimate of the parametric regressor for the behavioral response. We used the results of the one-sample t-test $(p=.05)$ reflecting activity modulated by reliving or understanding as an inclusive mask to determine whether the regions showing activation differences in self vs. other self-projection were also sensitive to behavior.

Task-Related Functional Connectivity Analysis-Seed voxels in the ventral vs. dorsal medial PFC that were identified in our previous analysis on self vs. other selfprojection were further interrogated to examine the task-related network of brain regions functionally connected with dissociable medial PFC regions. We should note that in the present article we refer to dorsal mPFC (z-axis on Talaraich atlas: $>20 \mathrm{~mm}$ ) and ventral mPFC (z-axis on Talaraich atlas: $<20 \mathrm{~mm}$ to $>-15 \mathrm{~mm}$; e.g., Krueger, et al., 2009; Van Overwalle, 2009), however, the particular naming convention may differ among authors (e.g., Buckner, Andrews-Hanna, \& Schacter, 2008; Northoff \& Bermpohl, 2004). To find these functional connectivity maps, we employed a second analysis based on individual trial activity (Rissman, Gazzaley, \& D'Esposito, 2004). Specifically, we first created a GLM in which each individual trial was modeled by a separate covariate, thus yielding different parameter estimates for each individual trial and for each individual subject. The resulting correlation maps were fisher transformed to allow for statistical comparison. Then, to examine differences in functional connectivity of ventral vs. dorsal medial PFC regions associated with temporal vs. mental self-projection we conducted a two-sample t-test in SPM5 using an FDR corrected threshold of $\mathrm{P}=.05$, and 2 voxel extent threshold.

\section{Results \\ Behavioral}

SPS was associated with a mean reliving rating of $5.04(S D=.56$; RT $=1.42 \mathrm{~s}, S D=.67)$, whereas SPO was associated with a mean understanding rating of $4.50(S D=.86 ; \mathrm{RT}=1.35$ $\mathrm{s}, S D=.61)$. There were no significant differences in the reaction time across the two conditions (Cohen's $d=.11$ ). The behavioral results suggest that the SenseCam images evoked a strong ability to re-experience the personal past and to comprehend another individual's perspective.

fMRI

Self-Projection of Self vs. Other-The results of the self vs. other self-projection revealed a dorsal vs. ventral distinction in the MPFC (see Table 1 and Figure 2). There was greater activity in the dorsal mPFC during SPO compared to SPS. Additionally, the SPO > SPS contrast revealed greater recruitment of right dorsolateral PFC and ventral parietal cortices. In contrast, there was greater recruitment of the ventral $\mathrm{mPFC}$ during self vs. other self-projection. Additionally, the SPS > SPO contrast revealed greater recruitment in several regions associated with AM retrieval (Cabeza \& St. Jacques, 2007), including the left hippocampus, lateral temporal, posterior midline and bilateral ventrolateral PFC. Interestingly, the difference between SPS vs. SPO was reflected by less deactivation in ventral $\mathrm{mPFC}$ but greater activity in dorsal $\mathrm{mPFC}$, which is a pattern of findings consistent 
with others (Gusnard, Akbudak, Shulman, \& Raichle, 2001) and linked to a default state of cognitive processing (Gusnard, Raichle, \& Raichle, 2001). In sum, these results suggest that ventral vs. dorsal subregions of the PFC contribute to separable forms of self-projection.

Parametric Modulation of Behavior-In order to directly link activation differences in self-projection to behavior, we examined activity in the above regions that was modulated on each trial by the extent of reliving in the case of SPS, and understanding in the case of SPO (see Table 1 and Figure 3). We found greater modulation of ventral mPFC during highly relived trials for SPS, but no modulation by understanding in this same region for SPO. In contrast, there was greater modulation of dorsal $\mathrm{mPFC}$ by better understanding for SPO, but no modulation in this region by reliving for SPS. In sum, these results show that activity in the ventral mPFC is sensitive to reliving when taking one's own past perspective during memory retrieval, whereas activity in dorsal mPFC is sensitive to better understanding when projecting one's self onto a different mental perspective.

Task-Related Functional Connectivity Analysis-For examining functional connectivity we used the peak voxels identified in the ventral and dorsal mPFC regions engaged by self vs. other self-projection as seed voxels in individual trial-based analyses. These analyses revealed that ventral vs. dorsal mPFC showed task-related functional connectivity with MTL and Frontoparietal Networks (see Table 3 and Figure 4). Ventral $\mathrm{mPFC}$ showed greater coactivation with the left hippocampus and precuneus, compared to dorsal mPFC. In contrast, dorsal mPFC showed greater coactivation with bilateral frontal and parietal regions, compared to ventral $\mathrm{mPFC}$. The pattern of functional connectivity of these ventral vs. dorsal mPFC regions is consistent with previous studies examining spontaneous patterns of coherent activity during passive resting state, which have revealed an MTL network (Kahn, et al., 2008; Vincent et al., 2006) and Frontoparietal Network (Vincent, et al., 2008). In sum, the findings from the functional connectivity analysis provide strong support for the dissociable role of dorsal vs. ventral mPFC in self vs. other self-projection.

\section{Discussion}

The present fMRI study investigated the neural mechanisms underlying self-projection to the personal past and into the life of another person within the same individuals via naturalistic stimuli that were prospectively generated using a novel camera technology. Our data indicate that there is a ventral vs. dorsal distinction in the $\mathrm{mPFC}$ during self-projection relying on shifts in perspective from one's own self vs. another individual. There were three main findings supporting this result. First, we found that temporal self-projection into the personal past recruited greater ventral $\mathrm{mPFC}$, whereas mental self-projection into another person's perspective recruited greater dorsal mPFC. Second, activity in ventral vs. dorsal $\mathrm{mPFC}$ was sensitive to parametric modulation on each trial by the ability to relive the personal past or to understand another's perspective. Third, task-related functional connectivity analysis revealed that ventral $\mathrm{mPFC}$ contributed to the medial temporal lobe network linked to memory processes, whereas dorsal mPFC contributed to the frontoparietal network linked to controlled processes.

MPFC is a critical node in the network of regions supporting tasks relying on self-projection such as AM retrieval (Buckner \& Carroll, 2007; Mitchell, 2009; Spreng, et al., 2009) and ToM (Buckner \& Carroll, 2007; Mitchell, 2009; Spreng, et al., 2009). Here we show that ventral vs. dorsal mPFC may differentially support self vs. other forms of self-projection during these tasks. The mPFC coordinates reported here also overlap with those found by a previous meta-analyses on social cognition (Van Overwalle, 2009) and one on selfreferential processing (Northoff, et al., 2006). The exact role of particular subregions within 
the mPFC has been a matter of considerable debate, with some studies observing recruitment of ventral mPFC when making inferences about one's own self and dorsal mPFC when mentalizing about others (Krueger, et al., 2009; Van Overwalle, 2009; also see Spreng, et al., 2009), and other studies observing that both dorsal and ventral mPFC recruited during self-referential processes (Northoff, et al., 2006). The recruitment of particular subregions of the mPFC during self-projection may vary according to the degree of personal relevance. Consistent with this idea, in a series of studies Mitchell and colleagues (Ames, Jenkins, Banaji, \& Mitchell, 2008; Jenkins, Macrae, \& Mitchell, 2008; Mitchell, Banaji, \& Macrae, 2005; Mitchell, Macrae, \& Banaji, 2006) found that ventral $\mathrm{mPFC}$ was recruited to a greater extent when mentalizing about similar others (e.g., people with the same political beliefs), presumably because they could rely more on the retrieval of information from their own life. In contrast, dorsal $\mathrm{mPFC}$ was recruited more when making inferences about dissimilar others. In the case of the current study, we found dorsal mPFC for a dissimilar other and ventral mPFC for a very similar other- one's own past self. Two previous studies investigating self-projection within the same individuals also found greater recruitment of ventral mPFC for AM versus ToM, but they did not observe differences in the recruitment of the dorsal mPFC (Rabin, et al., 2009; Spreng \& Grady, 2009). Control over the use of the first-person perspective during self-projection (e.g., D'Argembeau et al., 2007), employment of naturalistic dynamic visuospatial cues and other methodological differences may potentially account for the observed difference between the current and previous studies.

The recruitment of the mPFC also modulated the extent of self-projection to the personal past or into the life of another person. Ventral $\mathrm{mPFC}$ was recruited to a greater extent when temporal self-projection involved greater re-experience of the personal past as measured by subjective ratings of the amount of reliving. These results are consistent with prior functional neuroimaging studies showing that the ventral $\mathrm{mPFC}$ is sensitive to the ability to re-experience the self in time during AM (Levine, et al., 2004; Maguire \& Mummery, 1999), and with patient studies showing that lesions with overlap here impair the subjective experience of re-experiencing the personal past (Wheeler \& Stuss, 2003). In contrast, dorsal $\mathrm{mPFC}$ was recruited to a greater extent when mental self-projection involved better understanding of another person's perspective. Dorsal mPFC is recruited during tasks that rely on evaluation (Northoff, et al., 2006) such as during impression formation of another individual (Mitchell, Neil Macrae, \& Banaji, 2005). The sensitivity of dorsal mPFC to better understanding of a dissimilar other may reflect increased reliance on rule-based strategies to infer an alternative perspective.

Ventral vs. dorsal mPFC were functionally connected to separate neural networks, which differentially contributed to self-projection. Ventral $\mathrm{mPFC}$ showed greater functional connectivity with the hippocampus and precuneus, a pattern of results consistent with the MTL network supporting memory (Kahn, et al., 2008; Vincent, et al., 2006), whereas dorsal $\mathrm{mPFC}$ showed greater functional connectivity with lateral frontal, frontopolar, and dorsal parietal cortices, a pattern of functional connectivity which is consistent with the frontoparietal network supporting controlled processes (Vincent, et al., 2008). The MTL network is a subsystem of the default network, the set of brain regions that are coactive during passive resting states and associated with internally directed processes such as memory (Buckner, et al., 2008). Greater involvement of the MTL network during selfprojection to the personal past is consistent with the idea that the ability to take one's own perspective may rely upon the recovery of memory details. In contrast, the frontoparietal network supports the initiation and flexible adjustment of controlled processes (Dosenbach, Fair, Cohen, Schlaggar, \& Petersen, 2008). Vincent et al. (2008) suggested that the frontoparietal network may contribute to the integration between externally directed attention and internally directed thought, given that it is anatomically juxtaposed between 
the dorsal attention and default networks. These types of controlled processing may be particularly important during self-projection into the life of another person, which potentially involves greater integration between an externally presented perspective and internally directed processes. The functional dissociation between the neural networks supporting self-projection observed in the present study is also consistent with evidence that AM and ToM are independent (Rosenbaum, Stuss, Levine, \& Tulving, 2007).

Although mPFC is frequently observed in tasks relying on self-projection (Spreng, et al., 2009) it is also one of the most frequent regions observed during emotional tasks (Phan, Wager, Taylor, \& Liberzon, 2002). Very few studies have controlled for both self-reference and emotion within the same individuals, thus it is difficult to definitively distinguish these processes (for a meta-analysis see Gilbert et al., 2006). However, one study found that ventral $\mathrm{mPFC}$ was sensitive to the self-relevance of stimuli irrespective of emotion (Moran, Macrae, Heatherton, Wyland, \& Kelley, 2006). In the present study, the elicitation of emotional responses during self-projection was minimized, because the SenseCam images depicted very recent, everyday events (e.g., attending class, studying, etc.). Moreover, the dorsal vs. ventral distinction observed in the current study would be difficult to explain based on the role of these mPFC subregions in emotion (e.g., Dolcos, LaBar, \& Cabeza, 2004). Thus, the pattern of results observed in the present study is more consistent with the suggestion that the ventral vs. dorsal distinction in $\mathrm{mPFC}$ is related to differences in the nature of self-projection rather than emotion. Future studies, however, should directly manipulate the elicitation of emotion during self-projection for self vs. other.

\section{Conclusions}

The dynamic visuospatial cues employed in the current study provided a novel way to investigate self-projection of one's own life or the life of another individual. We found a ventral vs. dorsal distinction in the recruitment of the $\mathrm{mPFC}$ for self vs. other shifts in perspective. Further, ventral mPFC modulated the extent to which one's own perspective was re-experienced, whereas dorsal $\mathrm{mPFC}$ modulated the ability to understand an alternative perspective. Supporting the dissociable role of these $\mathrm{mPFC}$ subregions during different forms of self-projection, task-related functional connectivity analysis revealed that ventral vs. dorsal mPFC were nodes in different neural networks. Ventral mPFC contributed to the MTL network linked to memory processes, whereas dorsal mPFC contributed to the frontoparietal network linked to controlled processes. In sum, the results of the current study suggest that the mPFC contributes to shifts from the present moment to alternative self and other perspectives.

\section{Acknowledgments}

The authors would like to thank James A. Kragel for help with programming. This research was supported by a grant awarded to RC and MAC by Microsoft Research Cambridge and a National Institute of Aging RO1 (AG 23770) awarded to RC.

\section{References}

Ames DL, Jenkins AC, Banaji MR, Mitchell JP. Taking another person's perspective increases selfreferential neural processing. Psychol Sci. 2008; 19(7):642-644. [PubMed: 18727776]

Amodio DM, Frith CD. Meeting of minds: the medial frontal cortex and social cognition. Nat Rev Neurosci. 2006; 7(4):268-277. [PubMed: 16552413]

Brett M, Christoff K, Cusack R, Lancaster JL. Using the Talaraich atlas with the MNI template. Neuroimage. 2001; 13(6):S85.

Buckner RL, Andrews-Hanna JR, Schacter DL. The brain's default network: anatomy, function, and relevance to disease. Annals of the New York Academy of Science. 2008; 1124:1-38. 
Buckner RL, Carroll DC. Self-projection and the brain. Trends in Cognitive Science. 2007; 11(2):4957.

Cabeza R, Prince SE, Daselaar SM, Greenberg DL, Budde M, Dolcos F, et al. Brain activity during episodic retrieval of autobiographical and laboratory events: An fMRI study using a novel photo paradigm. Journal of Cognitive Neuroscience. 2004; 16(9):1583-1594. [PubMed: 15622612]

Cabeza R, St. Jacques PL. Functional neuroimaging of autobiographical memory. Trends Cogn Sci. 2007; 11(5):219-227. [PubMed: 17382578]

D'Argembeau A, Ruby P, Collette F, Degueldre C, Balteau E, Luxen A, et al. Distinct regions of the medial prefrontal cortex are associated with self-referential processing and perspective taking. J Cogn Neurosci. 2007; 19(6):935-944. [PubMed: 17536964]

Dolcos F, LaBar KS, Cabeza R. Dissociable effects of arousal and valence on prefrontal activity indexing emotional evaluation and subsequent memory: an event-related fMRI study. Neuroimage. 2004; 23(1):64-74. [PubMed: 15325353]

Dosenbach NU, Fair DA, Cohen AL, Schlaggar BL, Petersen SE. A dual-networks architecture of topdown control. Trends Cogn Sci. 2008; 12(3):99-105. [PubMed: 18262825]

Gallagher HL, Frith CD. Functional imaging of 'theory of mind'. Trends Cogn Sci. 2003; 7(2):77-83. [PubMed: 12584026]

Gilbert SJ, Spengler S, Simons JS, Steele JD, Lawrie SM, Frith CD, et al. Functional specialization within rostral prefrontal cortex (area 10): a meta-analysis. J Cogn Neurosci. 2006; 18(6):932-948. [PubMed: 16839301]

Gusnard DA, Akbudak E, Shulman GL, Raichle ME. Medial prefrontal cortex and self-referential mental activity: relation to a default mode of brain functio. Proc Natl Acad Sci U S A. 2001; 98(7): 4259-4264. [PubMed: 11259662]

Gusnard DA, Raichle ME, Raichle ME. Searching for a baseline: functional imaging and the resting human brain. Nat Rev Neurosci. 2001; 2(10):685-694. [PubMed: 11584306]

Jenkins AC, Macrae CN, Mitchell JP. Repetition suppression of ventromedial prefrontal activity during judgments of self and others. Proc Natl Acad Sci U S A. 2008; 105(11):4507-4512. [PubMed: 18347338]

Kahn I, Andrews-Hanna JR, Vincent JL, Snyder AZ, Buckner RL. Distinct cortical anatomy linked to subregions of the medial temporal lobe revealed by intrinsic functional connectivity. $\mathrm{J}$ Neurophysiol. 2008; 100(1):129-139. [PubMed: 18385483]

Krueger F, Barbey AK, Grafman J. The medial prefrontal cortex mediates social event knowledge. Trends Cogn Sci. 2009; 13(3):103-109. [PubMed: 19223228]

Levine B, Turner GR, Tisserand D, Hevenor SJ, Graham SJ, McIntosh AR. The functional neuroanatomy of episodic and semantic autobiographical remembering: a prospective functional MRI study. J Cogn Neurosci. 2004; 16(9):1633-1646. [PubMed: 15601525]

Maguire EA, Mummery CJ. Differential modulation of a common memory retrieval network revealed by positron emission tomography. Hippocampus. 1999; 9(1):54-61. [PubMed: 10088900]

Mitchell JP. Inferences about mental states. Philos Trans R Soc Lond B Biol Sci. 2009; 364(1521): 1309-1316. [PubMed: 19528012]

Mitchell JP, Banaji MR, Macrae CN. The link between social cognition and self-referential thought in the medial prefrontal cortex. J Cogn Neurosci. 2005; 17(8):1306-1315. [PubMed: 16197685]

Mitchell JP, Macrae CN, Banaji MR. Dissociable medial prefrontal contributions to judgments of similar and dissimilar others. Neuron. 2006; 50(4):655-663. [PubMed: 16701214]

Mitchell JP, Neil Macrae C, Banaji MR. Forming impressions of people versus inanimate objects: social-cognitive processing in the medial prefrontal cortex. Neuroimage. 2005; 26(1):251-257. [PubMed: 15862225]

Moran JM, Macrae CN, Heatherton TF, Wyland CL, Kelley WM. Neuroanatomical evidence for distinct cognitive and affective components of self. J Cogn Neurosci. 2006; 18(9):1586-1594. [PubMed: 16989558]

Northoff G, Bermpohl F. Cortical midline structures and the self. Trends Cogn Sci. 2004; 8(3):102107. [PubMed: 15301749] 
Northoff G, Heinzel A, de Greck M, Bermpohl F, Dobrowolny H, Panksepp J. Self-referential processing in our brain--a meta-analysis of imaging studies on the self. Neuroimage. 2006; 31(1): 440-457. [PubMed: 16466680]

Phan KL, Wager T, Taylor SF, Liberzon I. Functional neuroanatomy of emotion: a meta-analysis of emotion activation studies in PET and fMRI. Neuroimage. 2002; 16(2):331-348. [PubMed: 12030820]

Rabin JS, Gilboa A, Stuss DT, Mar RA, Rosenbaum RS. Common and Unique Neural Correlates of Autobiographical Memory and Theory of Mind. J Cogn Neurosci. 2009

Rissman J, Gazzaley A, D'Esposito M. Measuring functional connectivity during distinct stages of a cognitive task. Neuroimage. 2004; 23(2):752-763. [PubMed: 15488425]

Rosenbaum RS, Stuss DT, Levine B, Tulving E. Theory of mind is independent of episodic memory. Science. 2007; 318(5854):1257. [PubMed: 18033875]

Rubin DC, Burt CD, Fifield SJ. Experimental manipulations of the phenomenology of memory. Mem Cognit. 2003; 31(6):877-886.

Rubin DC, Schrauf RW, Greenberg DL. Stability in autobiographical memories. Memory. 2004; 12(6):715-721. [PubMed: 15724360]

Spreng RN, Grady CL. Patterns of Brain Activity Supporting Autobiographical Memory, Prospection, and Theory-of-Mind and Their Relationship to the Default Mode Network. Journal of Cognitive Neuroscience. 2009

Spreng RN, Mar RA, Kim AS. The common neural basis of autobiographical memory, prospection, navigation, theory of mind, and the default mode: a quantitative meta-analysis. J Cogn Neurosci. 2009; 21(3):489-510. [PubMed: 18510452]

St Jacques PL, Conway MA, Cabeza R. Gender differences in autobiographical memory for everyday events: Retrieval elicited by SenseCam Images vs. Verbal Cues. 2010 Under Review.

St Jacques PL, Rubins DC, Labar KS, Cabeza R. The Short and Long of It: Neural Correlates of Temporal-order Memory for Autobiographical Events. J Cogn Neurosci. 2008; 20(7):1327-1341. [PubMed: 18284345]

Suddendorf T, Corballis MC. The evolution of foresight: What is mental time travel, and is it unique to humans? Behavioral and Brain Sciences. 2007; 30(3):299-313. discussion 313-251. [PubMed: 17963565]

Van Overwalle F. Social cognition and the brain: a meta-analysis. Hum Brain Mapp. 2009; 30(3):829858. [PubMed: 18381770]

Vincent JL, Kahn I, Snyder AZ, Raichle ME, Buckner RL. Evidence for a frontoparietal control system revealed by intrinsic functional connectivity. J Neurophysiol. 2008; 100(6):3328-3342. [PubMed: 18799601]

Vincent JL, Patel GH, Fox MD, Snyder AZ, Baker JT, Van Essen DC, et al. Intrinsic functional architecture in the anaesthetized monkey brain. Nature. 2007; 447(7140):83-86. [PubMed: 17476267]

Vincent JL, Snyder AZ, Fox MD, Shannon BJ, Andrews JR, Raichle ME, et al. Coherent spontaneous activity identifies a hippocampal-parietal memory network. J Neurophysiol. 2006; 96(6):35173531. [PubMed: 16899645]

Wheeler MA, Stuss DT. Remembering and knowing in patients with frontal lobe injuries. Cortex. 2003; 39(4-5):827-846. [PubMed: 14584555]

Wixted JT. Dual-process theory and signal-detection theory of recognition memory. Psychol Rev. 2007; 114(1):152-176. [PubMed: 17227185]

Yonelinas AP. The nature of recollection and familiarity: A review of 30 years of research. Journal of Memory and Language. 2002; 46:441-517. 
A
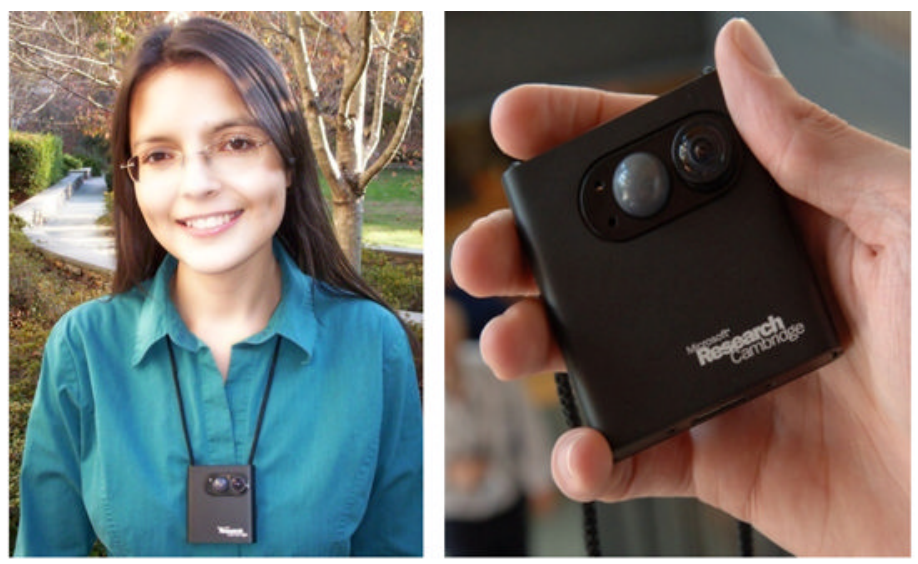

B
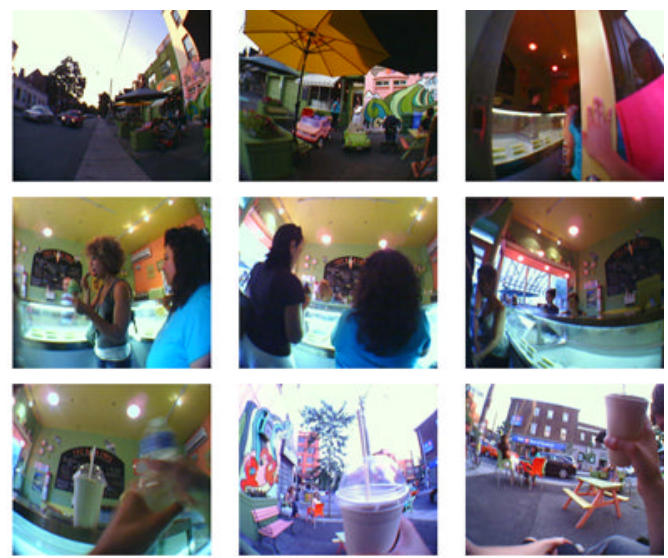

C

Self-Projection of one's own Self

(SPS)
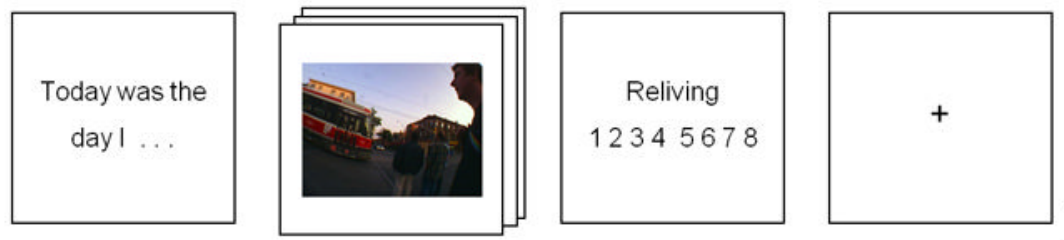

X12 per run

$10 \mathrm{~s}$

$20 \mathrm{~s}$

$6 s$

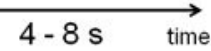

\section{Self-Projection of an} Other perspective

(SPO)
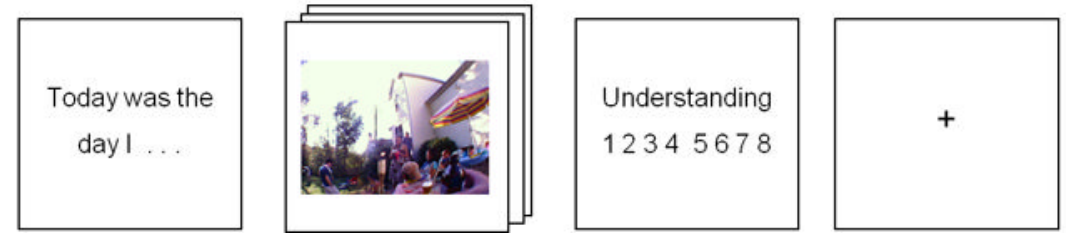

$\mathrm{X} 12$ per run

Figure 1. SenseCam

A) The SenseCam is a small wearable device that takes photographs automatically, without input from the user, B) SenseCam images acquired during a trip to the ice-cream shop, and C) The experimental design depicting the study conditions. 


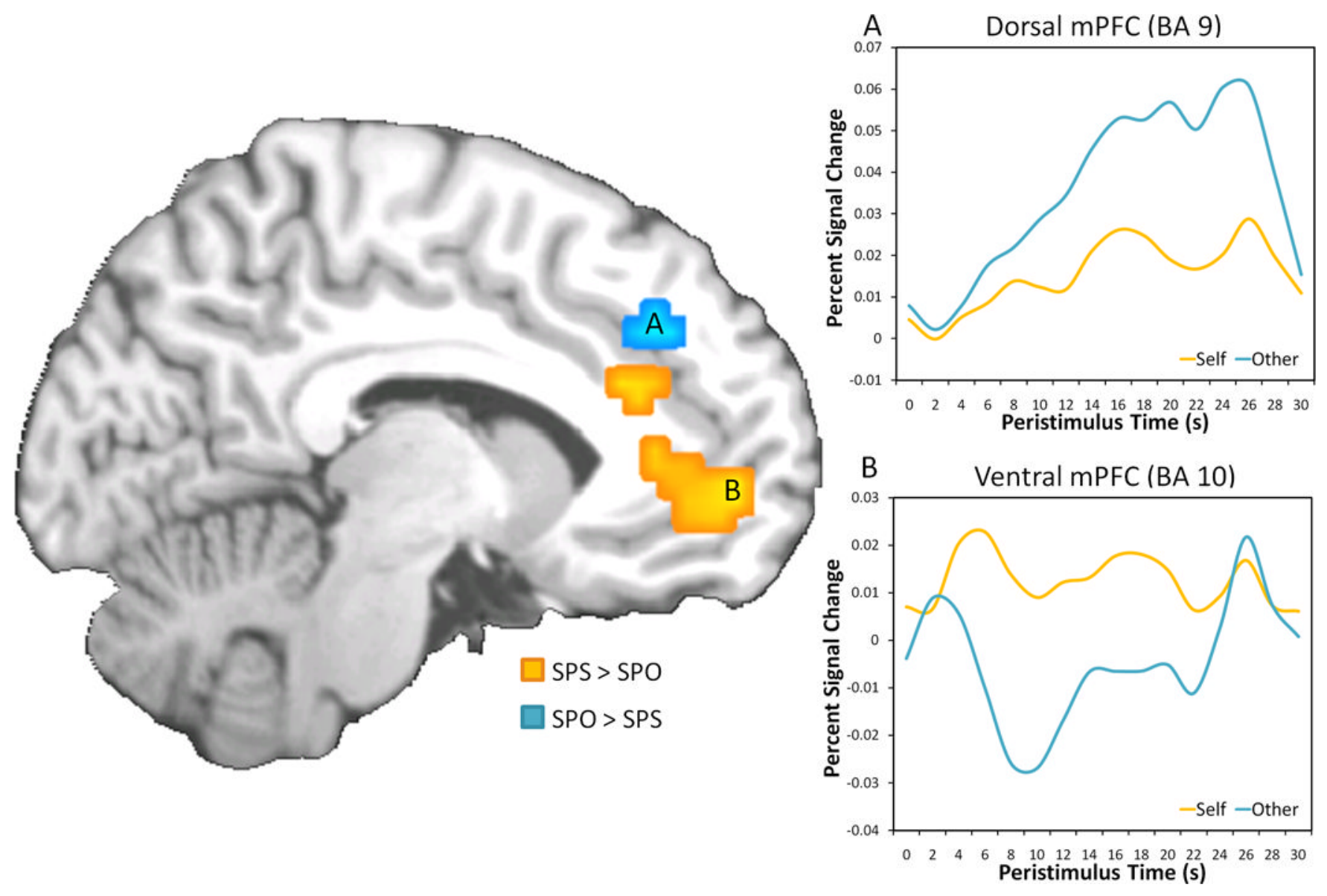

Figure 2. Self-Projection of Self vs. Other

There was a ventral vs. dorsal distinction in the recruitment of the medial prefrontal cortex (mPFC) during self vs. other self-projection. BA = Brodmann's Area. 

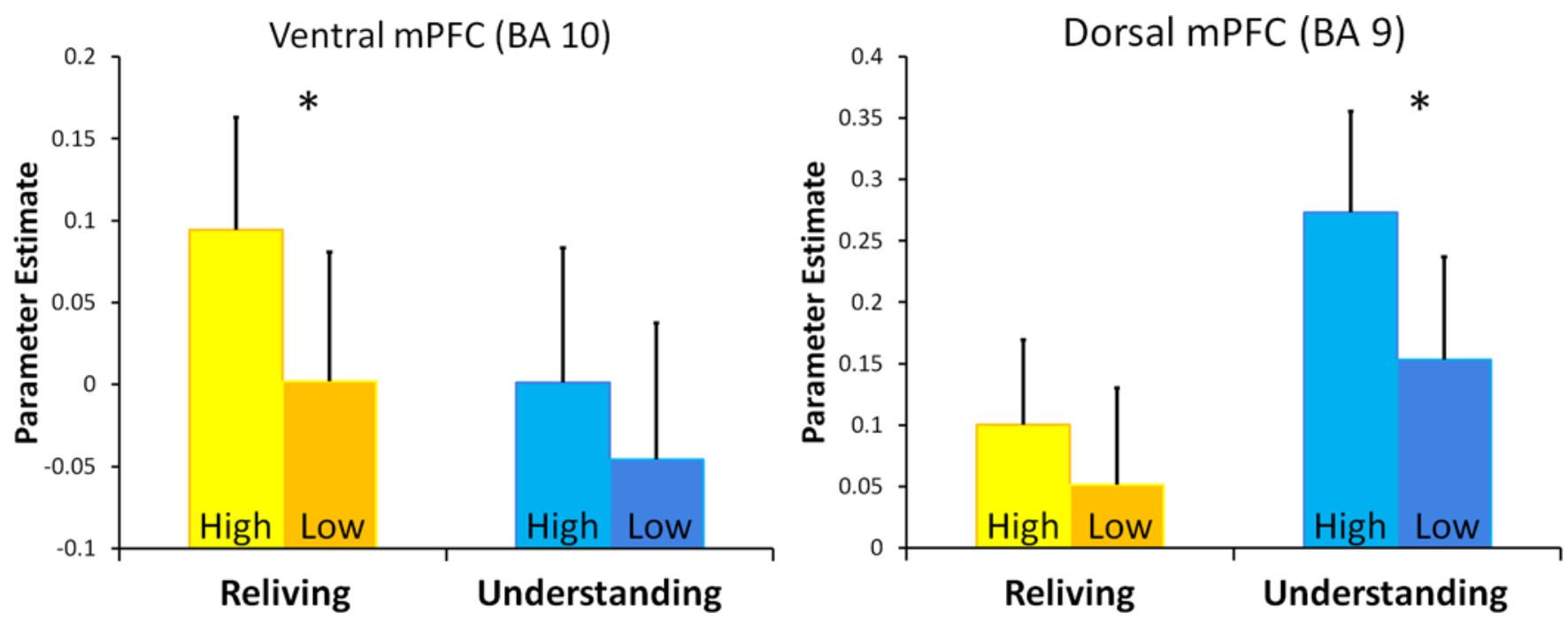

Figure 3. Parametric Modulation of Behavior

Ventral vs. dorsal medial prefrontal cortex (mPFC) modulated the amount of reliving during self-projection of one's own self (SPS) and the amount of understanding during selfprojection of another perspective (SPO), respectively. The graphs depict high and low ratings based on a median split; however, the parametric analysis is based on the continuous ratings. BA = Brodmann's Area. 


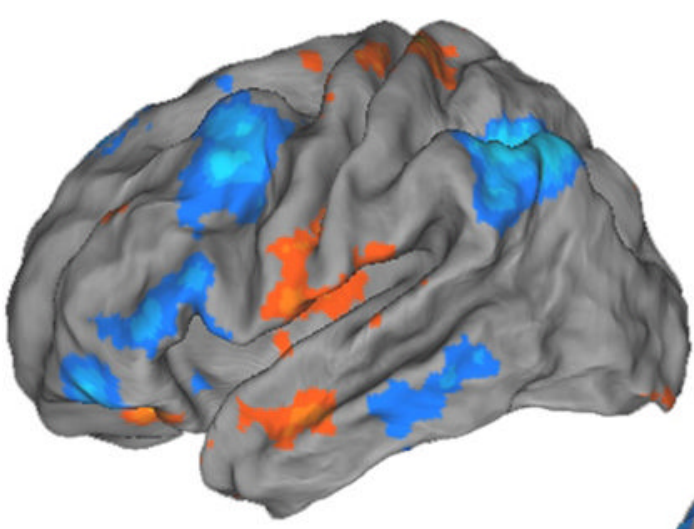

$-5.0-1.6 / 1.6 \quad 5.0$
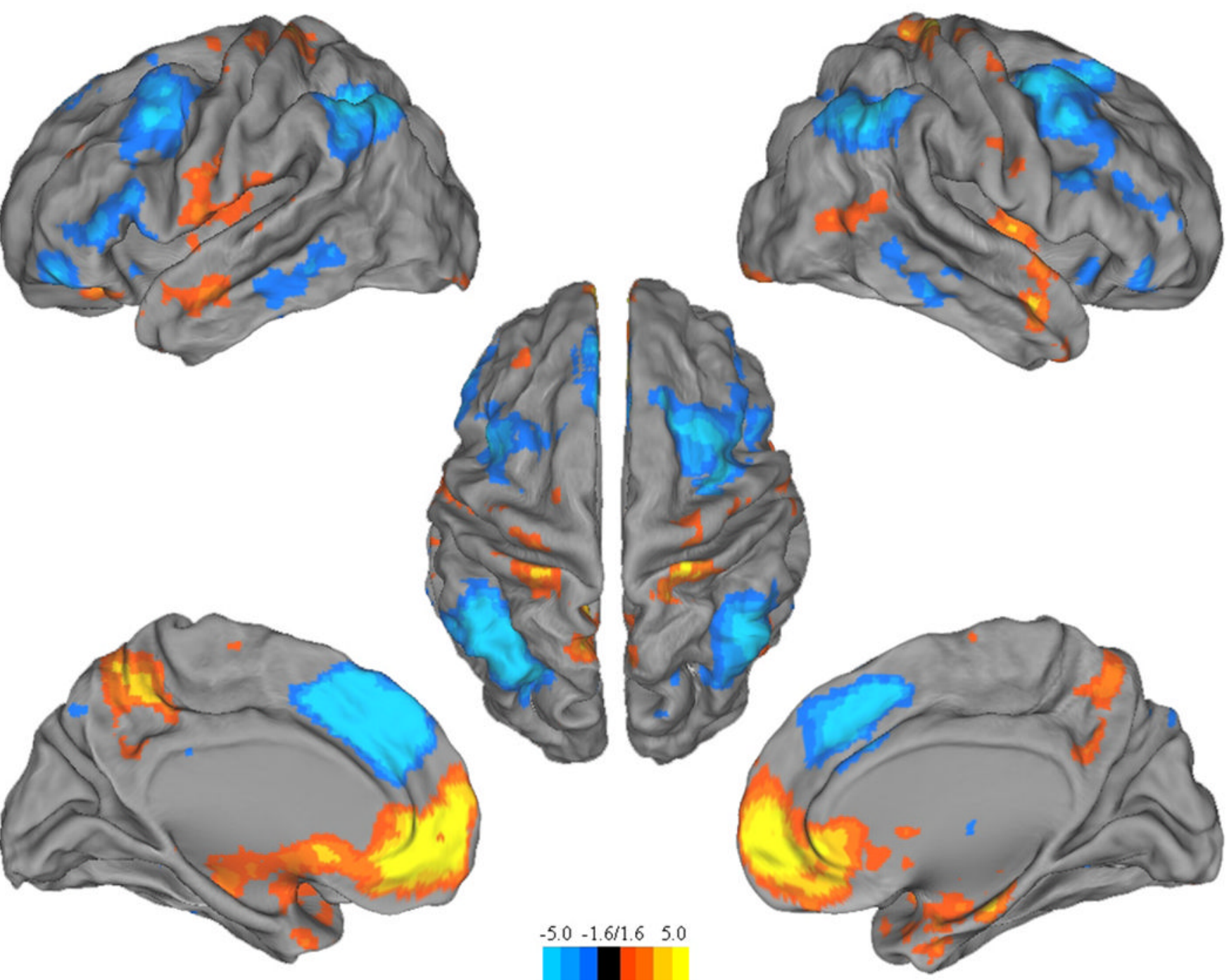

Figure 4. Task-Related Functional Connectivity Analysis

Ventral vs. dorsal medial medial prefrontal cortex (mPFC) were functionally connected to separate neural networks during self-projection of one's own self (SPS; orange/red activation) vs. self-projection of another perspective (SPO; blue activation). The activation in showing the results of the functional connectivity in each condition was projected to the cortical surface using CARET. 


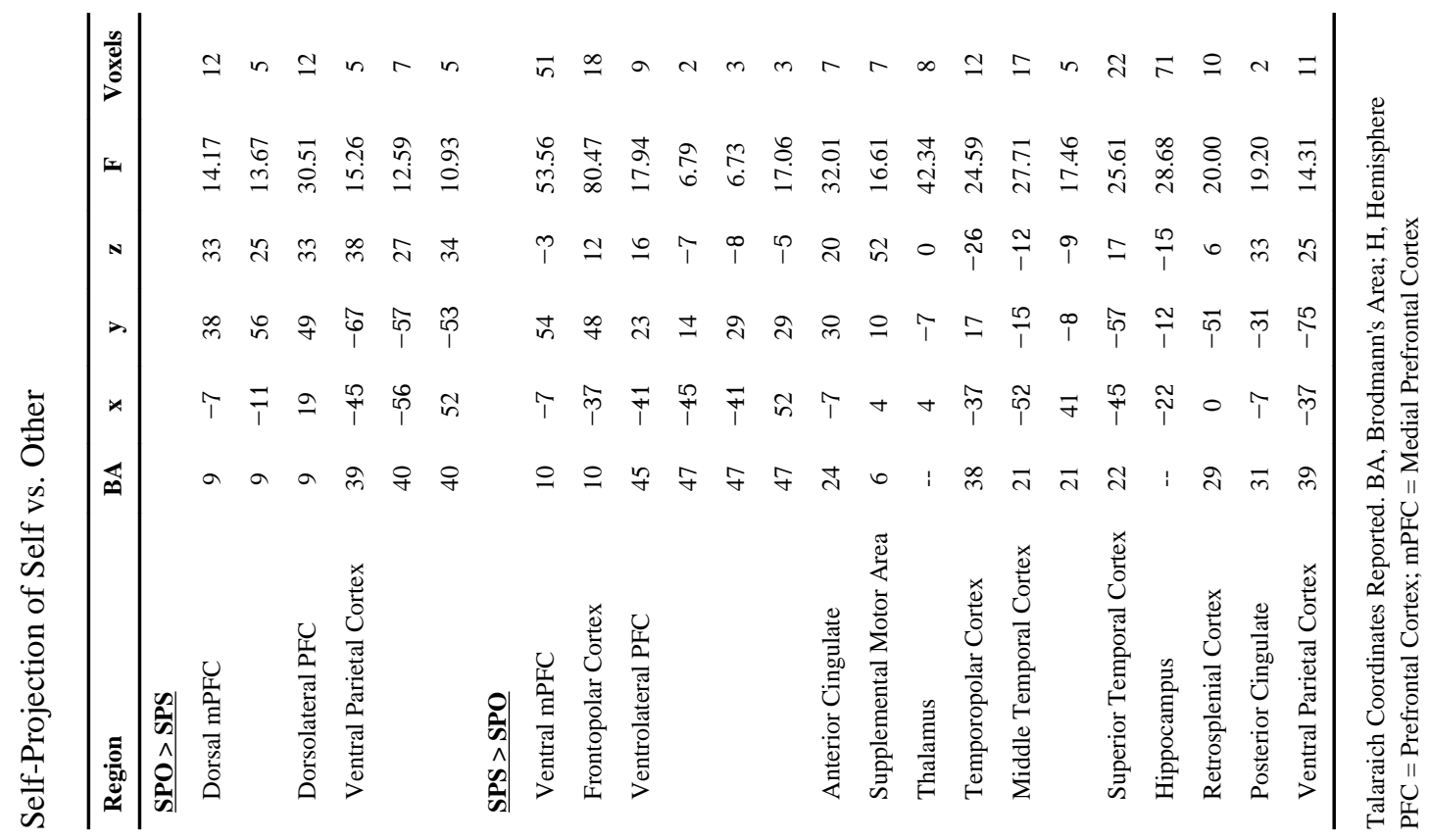




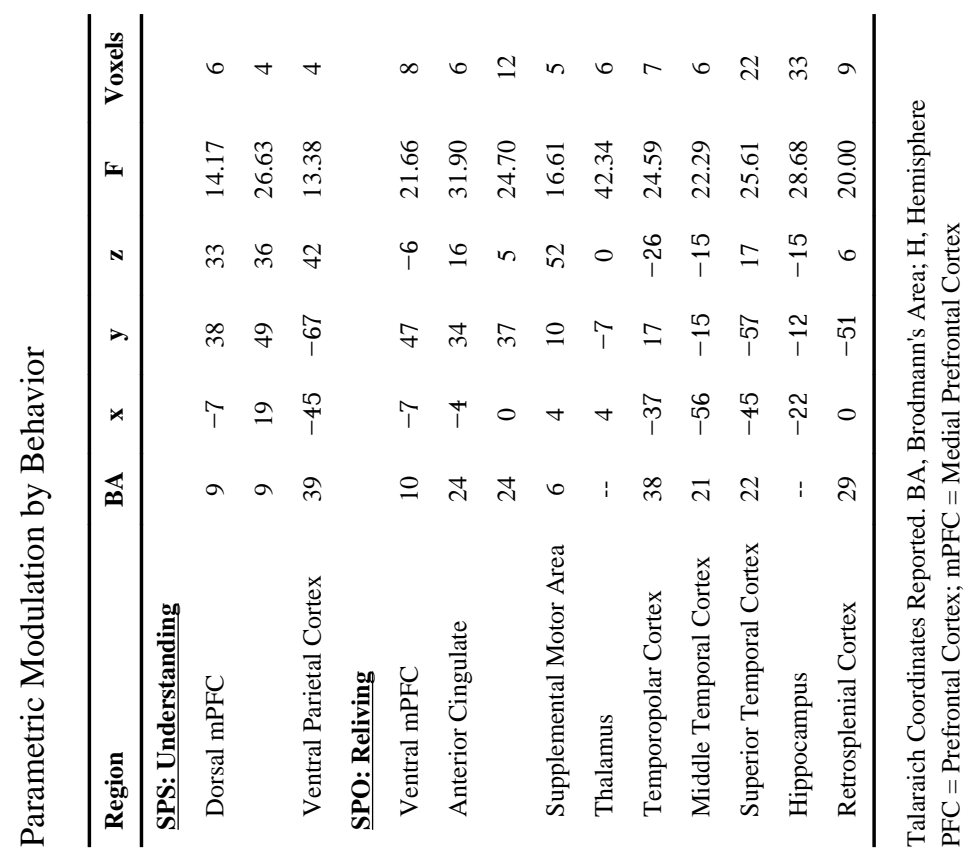
J Cogn Neurosci. Author manuscript; available in PMC 2012 June 1. 


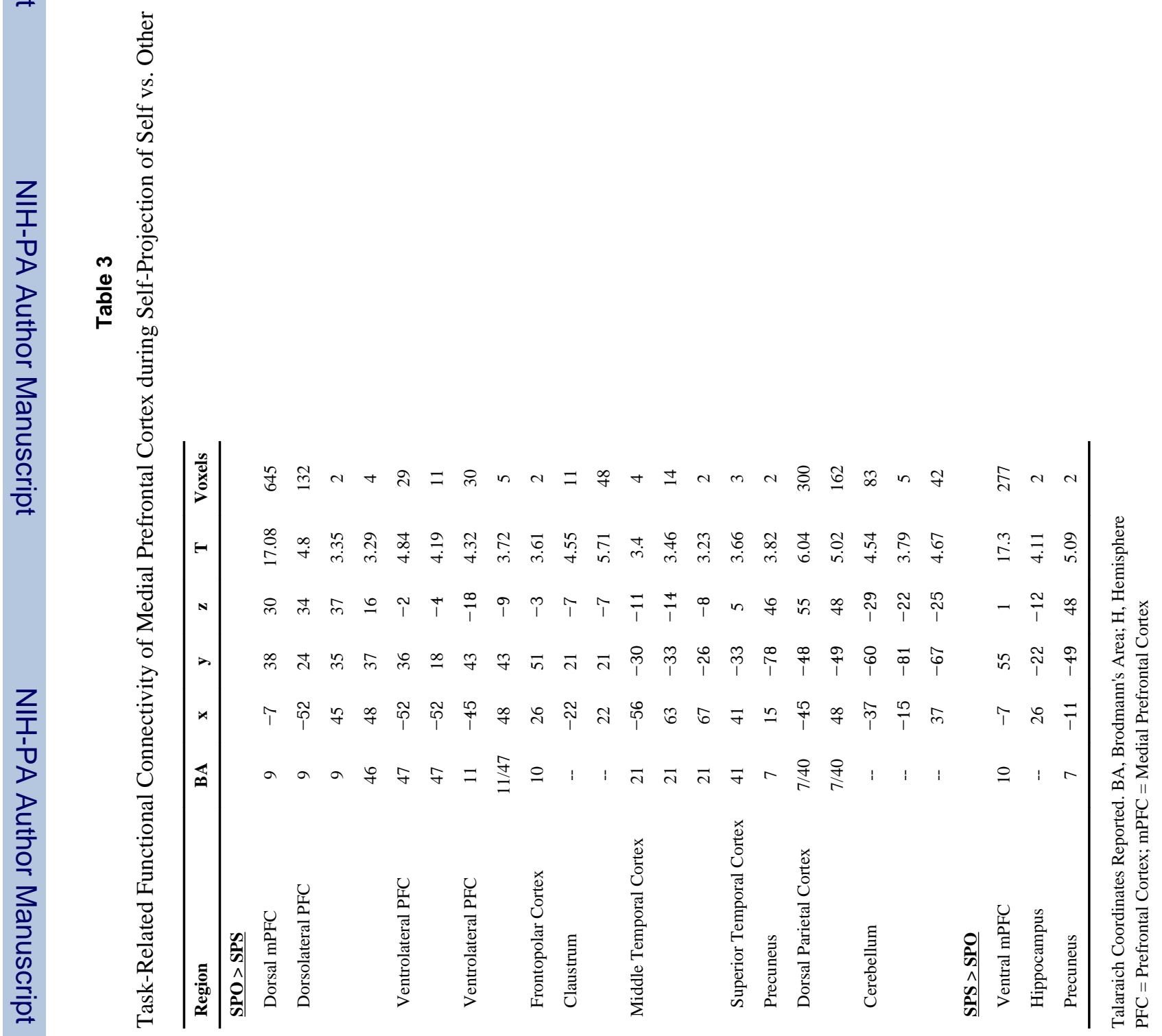

